\title{
Estudio cualitativo de los determinantes de la violencia escolar en Chile ${ }^{1}$
}

\author{
Mauricio García \\ Facultés Universitaires Saint Louis, Bruselas, Bélgica \\ Pablo Madriaza \\ Servicio de Salud Metropolitano Norte, COSAM de Conchalí, Santiago, Chile
}

\begin{abstract}
Resumen
Se presentan los resultados de una investigación cualitativa sobre violencia escolar realizada en Chile. En particular, se presentan los resultados relativos a los determinantes de la violencia escolar, según la percepción de estudiantes de enseñanza media que fueron considerados por sus instituciones educativas como agresivos o violentos. Dentro de la multiplicidad de aspectos que los jóvenes sitúan como determinantes de la violencia escolar, identificamos tres grandes categorías: (a) Antecedentes, que serían variables de contexto fuera del contexto específico de la violencia en la escuela; (b) Factores mediadores, que aluden a determinantes mediatos y, (c) Gatillantes, que se refieren a provocadores inmediatos, en particular de las peleas.
\end{abstract}

Palabras-clave: violencia escolar; determinantes; enseñanza media; percepción de estudiantes

\begin{abstract}
Qualitative study of school violence determinants in Chile. Results of a qualitative research on school violence carried out in Chile are presented. Particularly, this paper presents the results regarding the determinants of school violence according to the perception of high school students considered by their schools as aggressive or violent. Among the multiplicity of factors that young people establish as determinants of school violence, three broad categories are identified: (a) Antecedents, which would be contextual variables outside the specific context of violence in the school; (b) mediating factors, which refer to mediate determinants; and (c) triggers, which refer to immediate determinants, particularly of fights.
\end{abstract}

Keywords: school violence; determinants; high school; students’ perceptions

$\mathrm{E}$ n los últimos diez años, Chile ha visto emerger un fenómeno novedoso en las escuelas: la violencia. Durante mucho tiempo, se pensaba que los establecimientos educacionales estaban en cierta forma inoculados por una contaminación agresiva que se veía ya fuerte en la sociedad contemporánea. Un hecho de sangre inaugura mediáticamente el concepto de “violencia escolar” en Chile el año 1995 (Zerón, 2004), en plena transición democrática: un joven es muerto dentro de un establecimiento educacional.

Desde la ilustración, la escuela ha sido considerada un espacio de progreso y desarrollo, y en cierta forma, un espacio protegido. Dicho carácter que le ha permitido ocupar un papel fundamental dentro de la inserción social, y ha hecho de ella una suerte de abrigo o cortafuego para muchas de las desigualdades y horrores sociales. De ahí que resulte ciertamente desconcertante, pensar la posibilidad de la escuela como un espacio vulnerable. De ahí también que dentro de las violencias sociales, la violencia escolar sea una de aquellas consideradas más insensata e inexplicable (García \& Madriaza, 2005a). ¿Cómo es posible entonces que se dé violencia en las escuelas? ¿Por qué un espacio que se pensaba de resguardo, termina cambiando de signo, para aparecer bajo el alero de la vulnerabilidad? ¿Qué incide finalmente en la formación de esta particular manifestación social?

Pese a la ya inmanejable cantidad de información, es poco lo que sabemos sobre violencia escolar: el problema en el mundo apenas lleva treinta años de problematización e investigación seria (Ortega \& Mora-Merchan, 1997). Con 
todo, el énfasis ha estado puesto en discernir el fenómeno a partir del reporte de las víctimas. Ero es necesario también aprehender el fenómeno la pregunta sino desde la perspectiva del sujeto y del agente de la violencia escolar: éste es justamente el portador de un saber social sobre el ejercicio de su violencia. Y como portador de este "saber" anónimo, es él quien puede responder muchas de las interrogantes sobre las cuales se instala este fenómeno.

De esta forma, nuestra pregunta, viene abierta de antemano. Y viene abierta, en el sentido que no intenta responderse de manera apriorística, sino que viene dada como categoría vacía, dispuesta a ser llenada de las diversas significaciones vehiculizadas por aquellos que participan del fenómeno.

Así, este artículo es un intento de responder a la pregunta sobre la determinación de la violencia escolar, a través de la voz del sujeto de la violencia escolar. Es decir, a través de estudiantes que fueron considerados por sus instituciones educativas o por sus pares, como agresivos o violentos, y que así mismo, han participado en hechos que fueron considerados violentos por la escuela.

Los resultados que se presentan a continuación son reflejo de una parte de los resultados obtenidos en la investigación, que utilizó una metodología cualitativa para el análisis de entrevistas en profundidad y grupos focales de este tipo de estudiantes.

\section{Antecedentes: el problema de la definición de la violencia escolar}

Sin duda este es el gran problema que atraviesa la investigación en violencia escolar actualmente. Variados investigadores han hecho hincapié en la falta de acuerdo que existe sobre el tema (Colvin, Tobin, Beard, Hagan, \& Sprague, 1998; Defensor del Pueblo, 1999; García \& Madriaza, 2004; 2005a; Ortega, 1998). La pregunta en cuestión puede plantearse de esta forma: ¿Hasta que punto un acto puede ser considerado violento? ¿En que punto el acto llega a un nivel que necesita efectivamente ser detenido? El asunto en definitiva es un problema de límites no solo conceptuales sino también éticos. Dubet (en Zeron, 2004), por ejemplo, ve en toda esta explosión de preocupación social por la violencia escolar, un concepto ambiguo, "una categoría general que representa un conjunto de fenómenos heterogéneos, un conjunto de signos de dificultades de la escuela” (p. 4). Definición que resalta el amplio espectro de conductas que entran bajo el toldo del concepto y la multiplicidad de factores que confluyen en su generación, dando lugar no solo a formas de violencia escolar sino también a una violencia antiescuela (Dubet \& Martuccelli, 1998).

La literatura ha resaltado principalmente la violencia que se da entre estudiantes. En las primeras investigaciones hechas en países nórdicos el concepto que predominó fue el de mobbing, concepto que pese a ir en una línea bastante similar al bullying, pone el acento en las conductas de acoso y amenaza entre estudiantes (Olweus, 1998). Luego a través de la influencia de investigaciones desde USA, predominó el segundo concepto. Bullying traducido al español puede ser interpretado como matonaje, a un uso impropio y persistente de la fuerza por parte de un estudiante hacia otro. Olweus, por ejemplo, definió la violencia escolar en estos términos: "un alumno es agredido o se convierte en victima cuando está expuesto de forma repetida y durante un tiempo, a acciones negativas que lleva a cabo otro alumno o varios de ellos" (p. 25).

Las ideas sobre violencia en el nivel estudiantil, han sido fuertemente influenciadas por esta unilateralidad del uso del poder y la fuerza y por tanto, por la asimetría o desigualdad en el uso de éstas (Aron, 2000; Magenzo, Toledo, \& Rosenfeld, 2004; Pikas, 1989) de esta forma, la intimidación ha acaparado mucha de la investigación. En relación a esto, Magenzo et al. (2004) definen la intimidación como "el hostigamiento, el acoso, la amenaza sistemática que un estudiante o un grupo de estudiantes realiza a otro estudiante hombre o mujer” (p. 11). No es menor entonces, que Olweus (1998) entienda que el factor de riesgo más relevante a la hora de comprender la violencia entre estudiantes, sea el desarrollo físico. Sin embargo cuando se le pregunta a los estudiantes cuál es el icono más representativo de la violencia escolar, lo que aparece no es la intimidación, sino más bien la pelea, fenómeno que al contrario del primero, resalta cierta simetría en la relaciones (Debarbieux, 1996; García \& Madriaza, 2005a).

\section{Prevalencia}

Dado la anterior, se hace difícil confiar en la prevalencia. Existe una multitud de metodologías, instrumentos y operacionalizaciones que hace difícil esta tarea. En Latinoamérica las investigaciones sobre el tema han tardado en llegar y muchas de éstas, son aproximaciones indirectas a través de datos estadísticos no relacionados directamente con la violencia escolar. En Brasil, por ejemplo, el $13 \%$ de los estudiantes reportó que tanto estudiantes como profesores usan armas al interior de la escuela (Abramovay, 2003). En Uruguay, la percepción de violencia parece estar más acentuada: el $28 \%$ de los estudiantes reconoce haber participado en peleas y el $71 \%$ dice haber sido agredido verbalmente (Viscardi, 2003). En Ecuador, el 26 \% de los niños entre 6 y 10 años reconoce haber participado en una pelea y "el 36,7 \% de los adolescentes de 11 a 13 años han recibido y proporcionado golpes varias veces” (Maluf, Cevallos, \& Córdoba, 2003, p. 281).

En Chile, existen algunos estudios parciales (Lopez \& Filsecker, 2001; Magenzo \& Donoso, 2000; Navarro, 2003) y dos de ellos a nivel nacional (Gobierno de Chile, 2006a, 2006b; Instituto Ideas, 2005), que nos permiten por un lado ir dimensionando la situación de la violencia escolar y por otro, ver aquellos factores que pueden influir en el desarrollo de ella. En uno de estos estudios, el 34,4 \% de los estudiantes confesó que existe violencia de manera frecuente en sus establecimientos (todos los días + al menos una vez a la semana). Por otro lado, un 38,3\% reporta haber sido agredido alguna vez y un $8,1 \%$ lo ha sido de manera frecuente. Aquellos que confiesan haber agredido alguna vez, llegan al 36,8\% y un $8,4 \%$ confiesa haberlo hecho de manera frecuente (Gobierno de Chile, 2006a). 


\section{Comparación Chile/Brasil}

En un estudio de comparación internacional, utilizando información recolectada por el Observatorio Europeo de Violencia Escolar, y en base al mismo cuestionario, se pudo realizar una comparación entre diferentes países, entre ellos Chile y Brasil (Debarbieux, 2005; Gobierno de Chile, 2006b). Un dato que resalta es la percepción de violencia. En ella, el $12,4 \%$ de los estudiantes brasileños perciben que ésta se da de manera frecuente (siempre o casi siempre), mientras que en estas mismas alternativas, el porcentaje llega al 38,5\% entre los estudiantes chilenos.

Otras diferencias interesantes, tienen que ver en términos de agresión física, donde el 21,1\% de los chilenos reporta haber recibido este tipo de victimización, mientras que solo el 4,8\% de los brasileños hace lo propio. La situación se invierte, respecto a los robos con intimidación: el reporte de brasileños es casi cinco veces más alto que el de los chilenos $(\mathrm{Br}=29 \%$, $\mathrm{Ch}=5,7 \%)$.

\section{Factores involucrados}

Estudios en Chile han detectado algunos factores que podrían influir en la generación o disminución de violencia en la escuela (Contador, 2001; García \& Madriaza, 2004; Gobierno de Chile, 2006a; Llaña, 1999; Madriaza, 2005b; Madriaza \& García, 2005; Mena \& Vizcarra, 2001). Por un lado, el factor de género es relevante, así como el grado o curso en que se encuentran. Los hombres presentan consistentemente mayor participación en actos de violencia que las mujeres (Gobierno de Chile, 2006a; Madriaza \& García, 2005). Las mujeres tienden a emparejar con los hombres, solo en aquellas agresiones que son de corte psicológico (Gobierno de Chile, 2006a). En cursos de Enseñanza Básica existe un mayor reporte de agresiones que en cursos de Enseñanza Media, lo que indicaría que la edad puede ser un factor relevante (Contador, 2001; Gobierno de Chile, 2006a).

El nivel socioeconómico no parece ser un factor de diferencias, en términos brutos de violencia. En todos aparecen porcentajes relativamente similares. Las diferencias aparecen en términos del tipo de violencia ejercida. En niveles socioeconómicos más pudientes predominan las agresiones verbales y psicológicas y en los menos pudientes, predominan las agresiones físicas y delictuales (Gobierno de Chile, 2006a).

El clima escolar y la convivencia, son factores que son revelados como fundamentales a la hora de explicar, la formación o disminución de violencia (Mena \& Vizcarra, 2001).
Por ejemplo, en un estudio se encontró una correlación inversamente proporcional entre un índice de violencia y uno de clima escolar ( $r=-0,40$; Gobierno de Chile, 2006a). Lo que indicaría que mejorar el clima escolar, es un modo de influir en la disminución de violencia en la escuela. Por otro lado, la percepción negativa que existe entre estudiantes y profesores, es también un factor que influiría en un mayor conflicto entre ambos (Llaña, 1999).

García e Madriaza (2004, 2005a, 2005c, 2005d) han puesto de relieve en varias publicaciones la relevancia del testigo, como fuente de incentivo en la generación de actos de violencia. El agresor, sería un sujeto en permanente busca de reconocimiento de parte de sus pares, y su violencia sería un modo de ser reconocido positivamente por este testigo. El agresor cree que el testigo lo reconoce positivamente, a través de admiración o el temor, en la medida que él se violenta. Del mismo modo, en aquellos sujetos que habiendo sido agresores han dejado de serlo, un factor, que aparece como relevante en este cambio, es justamente la disminución de la necesidad de reconocimiento (García \& Madriaza, 2005d).

Por último, la salud mental aparece también como un factor a ser tomado en cuenta. En un estudio se muestra que los agresores son quienes muestran mayor presencia de sintomatología en salud mental, aún más que las víctimas (Madriaza, 2005b).

Si bien estos datos son suficientemente explícitos en cuanto a mostrar la existencia de violencia escolar en variadas formas en los países aludidos, no se cuenta con datos comparativos en el tiempo, que permitan evaluar si el fenómeno aumenta, ni tampoco con el significado de estos fenómenos de violencia registrados. Es a esta última tarea que quiere responder parcialmente esta contribución.

\section{Método}

\section{Participantes}

La muestra seleccionada fueron 64 estudiantes de enseñanza media (Secundaria) de Chile. La distribución por sexo fue de 12 mujeres y 52 hombres. La edad fluctuaba entre los 14 y 21 años, y se distribuían entre los diversos niveles de enseñanza ( $1^{\underline{0}}$ a $4^{\underline{0}}$ medio). De estos, 24 pertenecen a un nivel socioeconómico (NSE) Bajo, 20 al NSE alto y 20 al NSE medio.

El criterio de selección utilizado se basó en que estos estudiantes hayan sido señalados por su institución educativa

Tabla 1

Tipos de victimización recibida: Chile e Brasil

\begin{tabular}{lcccc}
\hline Tipo agresión & $\begin{array}{c}\text { Víctimas de robos } \\
\text { con amenaza }\end{array}$ & $\begin{array}{c}\text { Víctimas de } \\
\text { agresión física }\end{array}$ & $\begin{array}{c}\text { Víctimas de } \\
\text { insulto }\end{array}$ & $\begin{array}{c}\text { Víctimas de } \\
\text { insulto racista }\end{array}$ \\
\hline Brasil & 29,0 & 4,8 & 60,0 & 9,0 \\
Chile & 5,7 & 21,1 & 60,0 & 13,9 \\
\hline
\end{tabular}

Fuente: Debarbieux, 2005; Gobierno de Chile, 2006b 
y/o sus compañeros como agresivos o violentos; y que del mismo modo, hayan participado en hechos de violencia en la escuela. Siguiendo el estilo de selección, estos hechos de violencia fueron calificados como tales por el propio estudiante, sus compañeros y/o la institución.

Todos estos informantes son alumnos regulares de 11 establecimientos educacionales del país. De ellos, 3 son establecimientos de NSE alto, 4 de NSE medio y 4 de NSE bajo.

De esta forma, se completaron 54 entrevistas individuales y 27 grupos focales.

En aquellos informantes donde se vio que el material que presentaban, era mucho más rico y denso para los efectos de comprender el fenómeno, se decidió hacer una segunda entrevista, para profundizar en elementos que solo fueron superficialmente tratados en la anterior. Lo mismo ocurrió para los grupos focales, donde la mayoría fue citada para una segunda reunión y en algunos casos relevantes, incluso a una tercera.

\section{Técnica de recolección de datos}

La información fue recolectada por medio de dos dispositivos de carácter cualitativo: a través de la entrevista comprensiva y por medio de grupos focales. La entrevista comprensiva es una técnica que trata de vislumbrar el sistema de valores, elecciones sociales y lingüísticas que hace el informante y de adherir (sin exceso), con el fin de "liberar" el discurso y reforzar su sinceridad (Kaufmann, 1996).

Los grupos focales, corresponden a una conversación grupal, basada en la interacción de los participantes, aproximadamente 5 a 8 personas, focalizado en un tema propuesto por un facilitador (Taylor \& Bogdan, 1986).

Se optó por estos dispositivos de investigación cualitativa, porque privilegian un acceso más comprensivo a las representaciones que los jóvenes tienen de la violencia escolar. Es, en dichas representaciones donde podemos encontrar el sentido que ellos dan a este fenómeno. De esta forma, se propuso un abordaje combinado de recolección de datos, pues estos dispositivos, son dos contextos de interlocución donde las representaciones emergen de manera distinta.

\section{Procedimiento}

El primer paso fue hacer una invitación extensiva a participar de la investigación, a un número considerable de colegios preseleccionados por las características expuestas más arriba. De aquellos que acogieron esta invitación, se prefirió en primera instancia, a aquellos que fueran más representativos de los diferentes niveles socioeconómicos. De esta manera, la pretensión inicial de los investigadores no fue seleccionar establecimientos que fueran reconocidos por sus problemas de violencia, sino a aquellos representativos de sus niveles socioeconómicos, donde se hayan presentados estos problemas y se hayan sentido sobrepasados por ellos.

El siguiente paso consistió en la búsqueda de informantes que cumplieran con los requisitos expuestos. Esto se hizo a través de entrevistas iniciales con directivos y docentes que propusieron una lista preliminar de posibles informantes, lo cual fue corroborado o desmentido para la muestra final, a través de entrevistas de selección con los compañeros de estos estudiantes o a través de estos mismos estudiantes previamente seleccionados. En estas entrevistas de selección, se discutió básicamente con los informantes preliminares su posible inclusión o exclusión en la muestra de la investigación. En algunos casos, incluso se tuvo que buscar en otros establecimientos nuevamente, siguiendo el procedimiento antes expuesto, hasta llegar a aquellos más significativos. Así, el número de colegios participantes llegó a 11 .

Posteriormente a lograda la selección final de informantes se llevó a cabo la primera fase de entrevistas comprensivas propiamente tal. Después de esta fase y en base al material de las entrevistas, se realizó en estos establecimientos la fase de grupos focales, donde se congregó a un número significativo de estudiantes. Muchos de ellos ya habían sido entrevistados previamente, pero en la mayoría de los casos solo fue un porcentaje menor del grupo. Los grupos focales fueron conformados siempre por estudiantes del mismo establecimiento educacional y por rangos de edad similares. Es decir, se prefirió reunir en un mismo grupo, estudiantes de $1^{\underline{0}}$ y $2^{\underline{0}}$ medio y en otro de $3^{\underline{0}}$ y $4^{\underline{0}}$ medio. Los demás criterios de inclusión son los mismos que los enunciados más arriba.

Con el material recogido y trascrito, se realizó el análisis preliminar de la información, por medio del modelo de análisis cualitativo de la Grounded Theory (Strauss \& Corbin, 1994). Este análisis preliminar, fue realizado por los mismos entrevistadores, esta vez en parejas, supervisados por un coordinador general. De esta forma, se codificaron los datos a través de tres instancias propuestas por el modelo: codificación abierta, axial y selectiva. La fase final de análisis, fue realizada por el investigador encargado y el coordinador, donde se cotejó el análisis preliminar y se lo estableció de manera definitiva.

\section{Resultados}

Distinguiremos aquí tres niveles o categorías de determinantes de la violencia (ver Figura 1): 1) El primero y más amplio, son los antecedentes, es decir factores que escapan al contexto específico de la violencia pero que sin embargo influyen en ella o eventualmente son presentados como "causas" o "factores contextuales amplios" por sus participantes. 2) El segundo nivel son los factores mediadores, que aluden a determinantes que no son directos, sino mediatos, y que están presentes en el contexto específico de la violencia escolar. 3) El tercer nivel son los gatillantes, nivel que engloba todos los provocadores directos (inmediatos) del fenómeno, particularmente de la pelea (los mismos informantes la señalan como la manifestación más representativa de la violencia escolar). Por supuesto, estas distinciones permiten ordenar el material, pero no es una clasificación rigurosa, puesto que de hecho muchos eventos son difíciles de situar como antecedentes, mediadores o gatillantes, $\mathrm{y}$ tienden a ubicarse significativamente en más de una categoría de manera simultanea o simplemente tienden a estar al límite de alguna, tomando características de otra. 


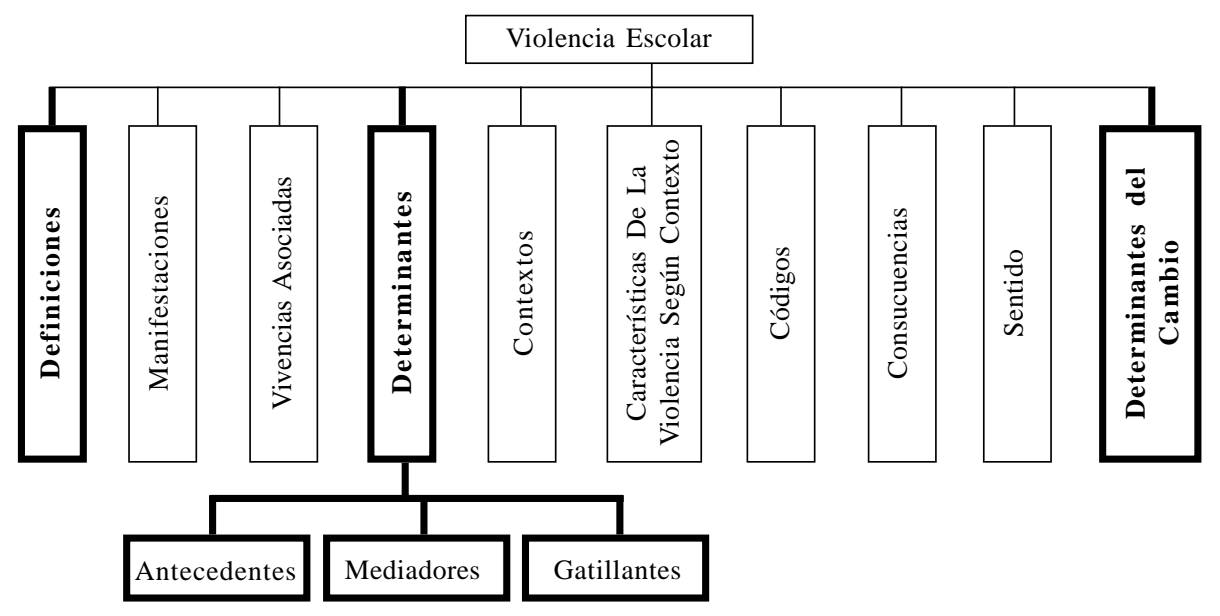

Figura 1. Estructura jerárquica de las dimensiones codificadas descriptivamente en la investigación.

\section{Factores antecedentes de la violencia escolar}

Distinguimos en este nivel tres dimensiones: a) Antecedentes individuales, b) Antecedentes familiares y c) Antecedentes socio-contextuales (Ver Figura 2).

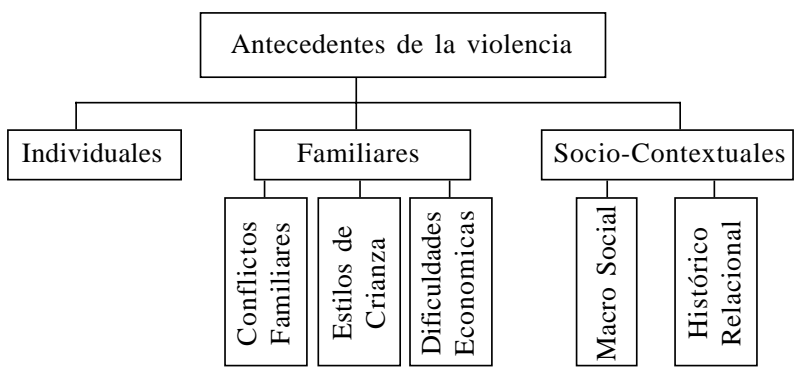

Figura 2. Árbol categorial de los antecedentes de la violencia.

a) Antecedentes individuales. La mayoría de los antecedentes individuales reportados aluden a dificultades psicológicas tales como: impulsividad, dificultad en relacionarse con los otros, dificultades emocionales (particularmente la rabia contenida), inmadurez y finalmente trastornos mentales. Respecto a la impulsividad, un joven nos dice en un grupo focal: "Sí es una cosa del momento, una cosa que uno no piensa, lo haces no más y cuando lo pensaste ya te mandaste la media cagada". Así, entre los mismos actores, tener poca capacidad de controlar los propios impulsos y darse cuenta de ellos, es un elemento significativo.

b) Antecedentes familiares. En esta dimensión se destaca la importancia de los conflictos familiares (separación de padres, por ejemplo), los estilos de crianza y las dificultades económicas. Los estilos de crianza, se basan en la idea de que ciertas familias han criado a sus hijos de un modo que ha promovido la resolución de conflictos de manera violenta "Porque los papás en las poblaciones" - nos cuenta, nuevamente Fernando, de 16 años - "a los niños los entrenan, desde chicos les enseñan a pelear. Entonces les dicen: ' $\mathrm{Y}$ cualquiera que se te pare, le pones un tajo no más'. Entonces andan con cortaplumas".

c) Antecedentes socio-contextuales. Al interior de esta categoría - desde los jóvenes -, se pueden distinguir dos clases de antecedentes. El primero es aquel que se refiere al plano de la historia relacional y el segundo más ligado a un nivel macrossocial.

Los antecedentes histórico-relacionales se refieren a una historia de interacciones con "pares o grupos", o con la "autoridad", que ha servido como base del desarrollo de violencia. Dentro de los pares o grupos aparecen las "amistades agresivas" y la "rivalidad histórica entre grupos" (grupos de pertenencia: pandillas, musicales, etc.). En un Liceo nos explican:

\begin{abstract}
al momento de elegir una especialidad, ya te formas una rivalidad mental contra la otra alianza: “que yo soy el más bacán [el mejor], los otros no me pueden ganar”. Entonces cuando uno siente como que ya le ganaron, se siente pica [encono] y entonces ahí queda la escoba. (Raúl, de 14 años)
\end{abstract}

En cuanto a las "relaciones con la autoridad" tienen importancia las dificultades que se han tenido con la ley o los agentes disciplinarios sociales (profesores, policía, etc.), sobre todo cuando los jóvenes han percibido sistemáticamente arbitrariedad o una actitud ofensiva de parte de estos a lo largo del tiempo. Los antecedentes macrossociales por el contrario trascienden lo grupal-relacional y afectan a la sociedad entera. Los más relevantes en el discurso de los jóvenes son el "individualismo social", el "stress del modo de vida", la "influencia de los massmedia", la "discriminación" y "mayor tolerancia social”. En relación a la "discriminación”, en un grupo focal se discute: "Si discriminan a todos los que son pobres, así no más es (Daniel). Si nos miran mal y eso mismo hacen que uno tenga ganas de pegarle (Manuel)". 
Particularmente saturados se encuentran los estigmas que pesan sobre el modo vestimentario, como marcador clave de la procedencia social según los jóvenes, y que da lugar a resentimientos, vivencias de humillación, lo que operaría como antesala de la violencia.

\section{Factores mediadores}

A modo general se puede decir que existen factores que median entre realizar o no una conducta violenta, es decir, algunos de ellos la facilitan mientras que otros la inhiben. En cualquiera de los dos casos éstos factores pueden ser tanto sociales - con una influencia indirecta -, como factores individuales, que se asocian más con características de la personalidad del joven y su mundo intrapsíquico (ver Figura 3).

a) Facilitadores de la violencia. Con respecto a los facilitadores sociales distinguiremos aquellos que son estrictamente relacionales, de aquellos que son situacionales. Los relacionales corresponden a elementos que surgen de las relaciones con individuos o grupos y que incitarían en algún grado el uso de la violencia. Los situacionales, por el contrario, corresponden a circunstancias con que el sujeto se encuentra, y que del mismo modo, facilitarían también el surgimiento de este tipo de fenómenos.

Dentro del ámbito socio-relacional, aparecen en el discurso de los jóvenes facilitadores tales como el "malentendido en la comunicación”, la "rivalidad con otros", las "provocaciones indirectas o sutiles”, el "abuso de poder" por parte de otro, el estar inserto en un "grupo curso o grupo de amigos desordenado”, el "ser etiquetado” o prejuzgado dentro de un cierto rol o estereotipo. Este último elemento es siempre importante y problemático para aquellos jóvenes que son definidos como conflictivos dentro de un establecimiento educacional. Por ejemplo Abel, de 17 años, relata que

sí llego a este colegio como un niño problema, cualquier cosa que pase se piensa que pude haber sido yo. Eso me da rabia y me han dado ganas de agarrarme a combos para que me dejen de lesear con eso.
La influencia del grupo curso se ve en la siguiente cita: "el curso tenía que hacerlo, porque era un grupo mayoritario, eran como 75 \% que hacía desorden, entonces los otros tenían que obedecer no más. Igual el curso influye caleta en una pelea o en una discusión”.

Respecto a los facilitadores socio-situacionales, aparecen con importancia para los jóvenes la "presencia” de - ciertos - “actores”, como neofascistas, carabineros, rivales desconocidos, etc. Leonardo de 16 años, atestigua en relación a esto último que "si estay peleando con alguien que no te conoce o que tú no conoces, nunca sabes cómo puede reaccionar y hay muchas más probabilidades de que se arme una pelea”. A veces la mera "presencia de otros" como testigos, puede ser un facilitador importante: “O sea, en relación a pelear, como que a uno le entusiasma que esté la gente...como que a uno lo pone con más energía”, nos cuenta Ariel de 17 años. También la “ausencia de autoridad”, incita en algún grado a la violencia.

Otras situaciones, esta vez ligadas al grupo curso, que facilitarían los hechos de violencia son, por ejemplo, el llegar a un "curso nuevo recién formado", o llegar como "alumno nuevo” a un curso antiguo. Luis, de 14 años, nos cuenta su experiencia en relación a esto: “pero aquí en un curso nuevo hay que hacerse respetar desde un principio, sino a uno lo van a agarrar para el leseo todo el año”. A esto se suma la importancia de un "curso que no delate" las acciones negativas, lo que podría funcionar también al modo de una ausencia de autoridad.

Pasemos ahora a revisar los facilitadores individuales, es decir características personales, relativas a cómo se es identidad - o como se está - estado -, y que en el discurso son presentadas como elementos que median en la implicación en la violencia escolar.

En relación a los aspectos de la identidad, aquellos que aparecen principalmente son el "ser agresivo", la "impulsividad" y lo que llaman algunos una "predisposición a la violencia", "creerse malo" o simplemente "ser choro" Estas últimas figuras, serían algunas modalidades de

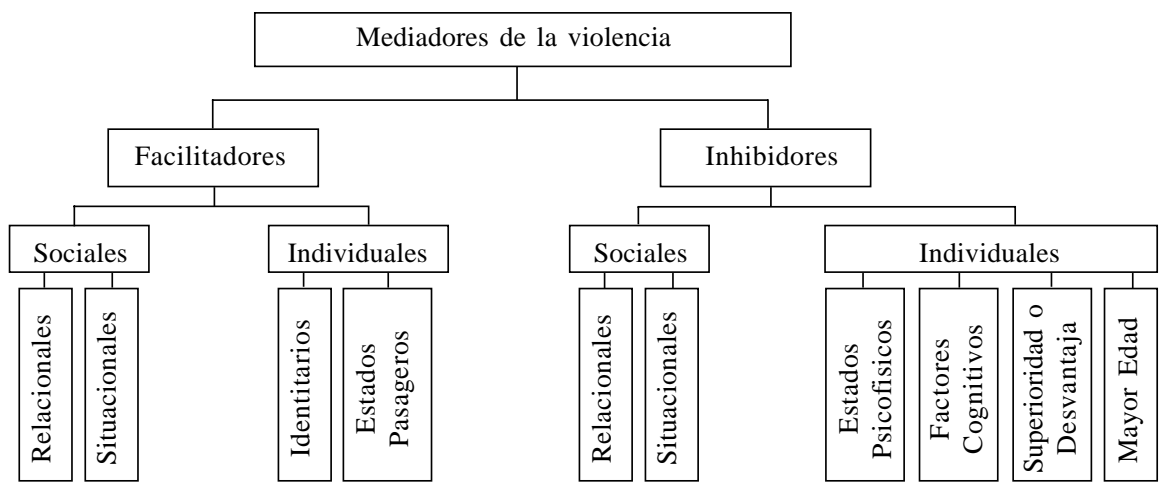

Figura 3. Árbol categorial de los mediadores de la violencia. 
identidad psicosocial y de rol, sobre todo en los niveles socioeconómicos más bajos, que para los jóvenes implican una actitud permanente de agresividad y desafío. "Porque son choros", nos explica Fernando, de 16 años, cuando le preguntamos por la violencia, "la juventud de ahora es chora. Yo también soy parado, soy choro". Otro joven nos explica que algunos estudiantes "se creen el cuento que son malos y andan con pistolas y... uno igual tiene que defenderse, si no te pasan a llevar...” (Nestor, 17 años). Por otro lado, el tener "problemas emocionales" (que podrían derivar de la familia quizá), y el ser de "menor edad”, resultan también ser facilitadores centrales según nuestros informantes. En relación a este último facilitador, es frecuente que sea asociado a mayor inmadurez, más impulsividad y desorden y a la capacidad de pelear por causas menos relevantes o francamente insignificantes.

En cuanto a los estados, el "acumular rabia" o "pica" [encono] y el "estar aproblemado" o "aburrido", son estados personales que frecuentemente son referidos como motores desde el cual pueden producirse actos violentos. Se suman los "estados alterados de conciencia", producto del consumo de alcohol y drogas que forman parte del espectro que presentan los informantes.

b) Inhibidores de la violencia. Al igual que en los factores facilitadores de la conducta violenta, esta categoría se articula en dos dimensiones fundamentales: el contexto social y el individual.

Los inhibidores sociales, siguiendo la misma clasificación de su antónimo facilitador, se dividen en sociorelacionales y en socio-situacionales. Entre los inhibidores socio-relacionales que fueron descritos por nuestros informantes, pudimos observar aquellos asociados a la "convivencia", por ejemplo conversar, desarrollar una amistad y también la familiaridad que se tenga entre los pares, así como la capacidad de pedir disculpas en situaciones en las cuales halla algún grado de responsabilidad personal. Otro inhibidor relacional importante es el "parentesco sanguíneo", es decir, se agrede mucho menos a personas de la familia del estudiante.

Entre los inhibidores socio-situacionales que perciben nuestros informantes es relevante la "presencia de terceros" (de autoridades, profesores, carabineros, etc.). Por ejemplo, una joven nos relata: "ayer no más casi se pusieron a pelear, pero la profesora habló con ellos y se calmaron”. También la presencia de otros significativos, como familiares o amigos, o cualquier intervención de terceros con la pretensión de inhibir, funciona amilanando las conductas violentas. El "desconocimiento" parece también ser un factor relevante a la hora de inhibir este tipo de conductas. Este "desconocimiento" puede estar asociado a un lugar físico, a un rival desconocido, o a un curso, al cual recién se llega como alumno nuevo. Por ejemplo: "si voy por un lado que no cacho [no conozco] y me están vacilando [molestando] a mi mina [novia], me voy a quedar tranquilo no más" (Gustavo, 16 años). Por último encontrarse en un "curso tranquilo" así como que el "rival no quiera seguir peleando", son elementos significativos para los estudiantes respecto a lo que desalienta el uso de la violencia.

En cuanto a los inhibidores individuales, agruparemos esta dimensión en cuatro categorías fundamentales: aquellos asociados a estados psicofísicos, a factores cognitivos, a la percepción de superioridad o desventaja personal y por último tener mayor edad.

Hay inhibidores individuales relativos a estados psicofísicos como el "cansancio" y el "sosiego", en el sentido de la vivencia asociada al período posterior a haberse "descargado" a través de la violencia. Esto es caracterizado por los informantes como una suerte de período refractario. Por ejemplo Pablo de 15 años, relata que "yo me había descargado por lo menos. El otro quería seguir, porque claro, le llegaron los combos. Pero, yo como salí ganador, no estaba ni ahí con seguir”.

Otros inhibidores de esta dimensión se asocian más bien a factores cognitivos, tales como la "capacidad reflexiva", la "inteligencia", la "toma de conciencia” y el "autocontrol". También se incluye la "capacidad de distraerse", es decir, la capacidad de pensar en otra cosa cuando surge cualquier forma de provocación o agresión y, por último, las "expectativas de consecuencias negativas", como la sanción familiar, escolar o social.

La percepción de desventaja o de superioridad personal respecto a un potencial rival, opera desalentando el paso a la violencia. Así por ejemplo, la "expectativa de daño posterior”, ya sea el daño que le hagan a uno o que se le haga al rival, la "percepción de una derrota inminente" en el curso de una pelea o "haber sido golpeado antes", parecen bloquear la conducta agresiva. Así mismo, "percibirse superior al rival", o "percibir el temor del oponente" también funcionan inhibiendo. Esto es lo que nos cuenta Rodrigo, de 17 años: "primero que nada, si yo sé que le pego, voy a tratar de calmar la cosa, para qué le voy a pegar si sé que le pego, porque yo cacho que tengo como más puntos a ganador".

Por último, un elemento muy importante que surge en el discurso de nuestros informantes son las características asociadas a tener mayor edad. Pues esto implicaría para ellos tener "mayor madurez", "mayor control de impulsos", "más tranquilidad" y "capacidad reflexiva” y "mayor respeto hacia el otro".

\section{Gatillantes de la pelea}

Con los gatillantes entramos al nivel más inmediato de la determinación de la violencia. Como los definimos al principio de este apartado, apuntan a aquellos actos o gestos que son considerados por los informantes como incitadores directos de un acto de violencia (generalmente la "pelea"). Es importante señalar, que no es el hecho en sí mismo el que incita la violencia, sino más bien la lectura del gesto. Vale decir, no siempre estos "gatillantes" van a desencadenar una pelea. Más bien esto va a depender de si el rival significa este mensaje como una provocación.

Hemos querido clasificar estos gatillantes, no ya por las características formales del evento, sino más bien, por la 
naturaleza de la afrenta. ¿Qué es lo que se transgrede en el sujeto, como para que se sienta incitado a reaccionar a través de la pelea? Así, como veremos estos se clasifican en 4 categorías:

a) Aquellos gatillantes que trasgreden la propia corporalidad o el espacio personal. Estas provocaciones van desde el que otro se acerque excesivamente, la obstrucción del paso o una actitud corporal de ataque, hasta la escalada de golpes en un juego rudo, el ser asaltado o las francas agresiones físicas. Las que tocan el espacio, son por ejemplo la invasión del territorio del espacio personal.

b) Aquellos que tocan las posesiones personales abarcan fundamentalmente el que alguien robe, sustraiga o dañe objetos personales.

c) Aquellos que implican una afrenta al honor y la respetabilidad. Primero están las afrentas que tocan a los otros significativos, acoso, humillación o agresión de seres queridos (pareja, amigos, conocidos o familiares). Algunas de estas afrentas al honor son de tipo más bien verbal, como por ejemplo, "las amenazas", los “palabreos” y "discusiones", el que alguien "levante falso testimonio", las "calumnias o cahuines", o que "se delate”. Se agregan las "bromas y burlas”, y las "groserías fuertes”. Otras tienen un carácter gestual, como por ejemplo “chasquear los dedos”, “trompear”, "mirar en menos" y "mirar feo". El "mirar feo" alude a que alguien mire al sujeto de manera insistente: este es un código, que en el discurso es presentado como un llamado a la violencia y a la pelea. El que sostiene la mirada y mira feo, buscaría demostrar su superioridad y su fortaleza. Frente a esto hay dos opciones: o se baja la mirada o se la sostiene. La primera implica sometimiento y la segunda desafío, y esta incidencia gatilla la pelea o la violencia.

d) Finalmente, aparecen con mucha relevancia el ser pasado a llevar y el hostigamiento. Este último concepto nos parece muy revelador, pues implica cualquier provocación que tome un carácter insistente y sistemático, y por lo tanto puede constituirse a partir de cualquier elemento de las categorías anteriores. El ser pasado a llevar alude a la falta de respeto de los otros, es decir afrentas en que el sujeto no se siente reconocido en cuanto tal. De no responder a esta afrenta serán luego objeto de sucesivas y permanentes agresiones y humillaciones. Así, desde estos códigos de la afrenta, el honor y el reconocimiento, la violencia es entendida frecuentemente como necesaria e inevitable.

\section{Conclusiones}

¿Cuál es el interés y el valor de estos resultados? La teoría de las representaciones sociales (Moscovici, 1961; Jodelet, 1989) nos ha enseñado que no son los factores “objetivables” los que determinan la conducta social, sino las representaciones subjetivas e intersubjetivas que los actores sociales construyen de dichos factores. El modelo que hemos construido, a través de antecedentes, mediadores y gatillantes, representa justamente la representación construida socialmente por los jóvenes en la convivencia escolar, de lo que efectivamente determina su violencia. En este sentido, el conjunto de distinciones propuestas, articulan una suerte de "gramática íntima” que da cuenta de los factores y dimensiones que operan de hecho como determinantes de la violencia.

Con todo, nuestra investigación previa (García \& Madriaza 2004, 2005a, 2005b, 2005c), nos sugiere que dichos factores, sobre todo los mediadores y los gatillantes, se articulan de manera sustantiva con una problemática nuclear en la violencia escolar, a saber, la necesidad de reconocimiento, necesidad de sentirse existente para el otro, validado socialmente, sea esto bajo la forma de ser amado, odiado, o temido. Una de las modalidades concretas más frecuentes en que se manifiesta esta necesidad es en el imperativo de ser respetado o “imponer el respeto”, a través de la violencia o la amenaza. En este sentido las contribuciones de Todorov (1995) y más recientemente de Le Breton (2002), son decisivas para entender la violencia escolar y la violencia juvenil.

La problemática del reconocimiento se presenta a dos niveles: a) en la relación diádica con el rival, en la situación paradigmática de la pelea; b) en las relaciones triádicas o grupales, done a la pelea entre dos (protagonista y antagonista) se le agrega al menos un testigo, que presencia la pelea o simplemente sabe de ella. En efecto, es el reconocimiento de los pares testigos que es buscado fundamentalmente en el recurso a la violencia.

Como decíamos al comienzo, la intimidación ha sido el fenómeno más estudiado en la investigación empírica de la violencia escolar. Ella supone ante todo, una relación asimétrica y sostenida en el tiempo, donde un agresor hostiga a una víctima. No obstante, el icono de la violencia escolar para estos jóvenes “agresores” en Chile, no es la intimidación (pese a que no se descarta) sino más bien, la “pelea”. En este punto concordamos con las investigaciones de Debarbieux (1996). La pelea, al contrario de la intimidación, supone cierta simetría en el ejercicio de la violencia. Recordemos que un factor inhibidor de ésta, es percibirse como superior o inferior al rival. De esta forma, la pelea, para tener sentido social, debe darse no contra una victima, sino más bien contra un igual. Suponer que el rival contra el cual se lucha es un igual, implica que la violencia es un acto de reconocimiento del antagonista y no necesariamente, una falta de consideración de éste. De esta forma, estos sujetos de la violencia escolar, no buscan destruir al rival, sino establecer una suerte de ritual del reconocimiento. La legalidad subterránea de la pelea supone la necesidad de ciertos imperativos en ella, por ejemplo, el que sea uno a uno: "Si un loco me dice 'ya, a la salida [peleamos]'. Yo, obviamente, voy a ir solo a la salida, y él también tiene que estar solo. Eso es la lealtad”. La inclusión de un tercero activo, supone siempre un acto vicioso que ensucia el ritual de reconocimiento: "si por ejemplo, un curso me amenaza a mi, yo voy a ir con mi curso a defenderme, porque un curso contra un curso tiene que ser más parejo".

Hablamos por cierto del reconocimiento del rival - es decir, un fenómeno de a dos -, donde el tercero, al intervenir, ensucia el ritual de la pelea. No obstante la presencia subjeti- 
va de este tercero es clave, pues su mirada es fundamental en la satisfacción de la necesidad de reconocimiento (García \& Madriaza, 2004, 2005d). El antagonista, al pelear, instituye al sujeto como un par, como alguien digno del ritual. Por su parte, el testigo, con su presencia, no implicándose activamente en la pelea, testimonia de su interés, incluso de su admiración o respeto por quienes pelean. Esto ocurre incluso cuando el testigo no "vio" la pelea, sino que solo "supo" de ella. Entre los gatillantes de la pelea puede observarse esta característica. Cuando hablamos del ataque al "honor y la respetabilidad", estamos incluyendo inevitablemente el carácter simbólico que adquiere el reconocimiento: ¿Qué es realmente lo que se ataca, cuando se desencadena la violencia? ¿Qué se indica con la palabra respeto y honor? No es ni siquiera el cuerpo material, cuando el objeto del ataque es la corporalidad. De ahí que la llamemos corporalidad y no simplemente cuerpo. La corporalidad es ante todo un cuerpo imaginado, un espacio y frontera subjetiva, cuyos límites pueden o no pueden estar en el límite material del cuerpo real. Es decir, lo único que tenemos, es que hay una frontera que es traicionada, subvertida y donde parece estar jugándose lo más propio e intimo del sujeto de la violencia: su imagen ante el otro. Este es el drama del reconocimiento. $\mathrm{Y}$ en cierta forma podemos concluir que este es el verdadero determinante de la violencia en las escuelas. Esta perspectiva torna la violencia escolar un fenómeno tan comprensible como cualquier otro evento social, y lo aleja del ámbito de lo in-sensato.

¿Qué hace entonces, que el reconocimiento en estos jóvenes se haya volcado hacia la violencia? ¿En que ha fallado el sistema escolar que no ha dado cabida a otras formas de reconocimiento que absorban esta sed social? Es imprescindible responder a esta necesidad de reconocimiento, pues la estrategia disciplinaria (más o menos rigurosa y fuerte) puede pretender a doblegar voluntades pero raramente formar sujetos. El que hay una necesidad de reconocimiento insatisfecha en los estudiantes violentos del nivel secundario en Chile, ha sido puesto en evidencia indirectamente por Leal, Meneses, Alarcón e Karmelic (2005) quienes aplicando una escala de resentimiento, encuentran que los alumnos que presentan comportamientos agresivos graves muestran significativamente más resentimiento que el resto de la muestra. Esperamos haber contribuido parcialmente a entregar elementos que permitan atender a las significaciones íntimas y sociales que fabrican los determinantes de la violencia escolar en Chile, y esperamos que otras investigaciones puedan profundizar, corregir o relativizar, por la vía comparativa, algunos de estos resultados.

\section{Referencias}

Abramovay, M. (2003). Enfrentando a violência nas escolas: um informe do Brasil. In M. Abramovay (Org.), Violência na escola. América Latina e Caribe (pp. 89-152). Brasília: UNESCO.

Aron, A. (2000). Un programa de educación para la no-violencia. Psykhe, 2(9), 25-39.
Colvin, G., Tobin, T., Beard, K., Hagan, S., \& Sprague, J. (1998). The school bully: assessing the problem, developing interventions, and future research directions. Journal of Behavioral Education, 3(8), 293-320.

Contador, M. (2001). Percepción de violencia escolar en estudiantes de enseñanza media. Psykhe, 1(10), 69-80.

Debarbieux, E. (1996). La violence en Milieu Scalaire. 1. État des Lieux. Paris: ESF.

Defensor del Pueblo. (1999). Informe del Defensor del Pueblo sobre Violencia Escolar. Madrid: Autor.

Dubet, F., \& Martuccelli, D. (1998). En la escuela: sociología de la experiencia escolar. Buenos Aires: Losada.

García, M., \& Madriaza, P. (2004). Clamores juveniles y su relación con la violencia escolar: conflicto y aspiración por recrear un orden social. Revista Persona y Sociedad, 18(1), 31-50.

García, M., \& Madriaza, P. (2005a). Lineamientos para la intervención en violencia escolar en Chile. Revista Persona y Sociedad, 19(1), 135-154.

García, M., \& Madriaza, P. (2005b). Sentido y propósito de la violencia escolar. Análisis estructural del discurso de estudiantes agresivos del nivel socioeconómico alto. Revista de Psicología, 14(1), 41-60.

García, M., \& Madriaza, P. (2005c). Sentido y sinsentido de la violencia escolar. Análisis cualitativo del discurso de estudiantes chilenos. Psykhe, 14(1), 165-180.

García, M., \& Madriaza, P. (2005d). La imagen herida y el drama del reconocimiento. Estudio cualitativo de los determinantes del cambio de la violencia escolar en Chile. Revista Estudios Pedagógicos, 31(2), 27-41.

Gobierno de Chile, Ministerio del Interior \& Ministerio de Educación (2006a). $1^{\circ}$ Estudio Nacional de Violencia en el Ámbito Escolar. Informe Encuesta Alumnos. Santiago: Autor.

Gobierno de Chile, Ministerio del Interior \& Ministerio de Educación (2006b). $1^{\underline{o}}$ Estudio Nacional de Violencia en el Ámbito Escolar. Informe Comparación Internacional. Santiago: Autor.

Instituto Idea Chile (2005). Estudio de convivencia escolar. La opinión de estudiantes y docentes. Santiago: Autor.

Jodelet, D. (1989). Les représentations sociales, Paris: Presses Universitaires de France.

Kaufmann, J-C. (1996). L'entretient compréhensif. Paris: Nathan.

Le Breton, D. (2002). Conduites à risque, Paris: Presses Universitaires de France.

Leal, F., Meneses, M., Alarcón, A., \& Karmelic, V. (2005). Agresión y resentimiento en los estudiantes secundarios. Revista de Psicología, 14(1), 29-40.

Llaña, M. (1999). La perspectiva del Sistema Educacional respecto a la violencia infanto-juvenil. Revista de Psicología, 2(8), 39-43.

Lopez, M., \& Filsecker, M. (2001). Manifestaciones de violencia y agresividad en la escuela: estudio comparativo en tres realidades geográficas distintas. Santiago: ICET.

Madriaza, P. (2005a). Poder/violencia y el ejercicio latinoamericano de dominación inconclusa. Revista Persona y Sociedad, 19(1), 249-262.

Madriaza, P. (2005b). Resultados descriptivos de estudio internacional sobre violencia y convivencia escolar en estudiantes de enseñanza media de la ciudad de Santiago de Chile. Comunicação apresentada em “ $2^{\mathrm{o}}$ Seminario de Violencia y Delincuencia”, organizado por Paz Ciudadana e Instituto de Sociología de la Pontificia universidad Católica de Chile, Santiago de Chile.

Madriaza, P., \& Garcìa, M. (2005). Resultados descriptivos de estudio internacional sobre violencia y convivencia escolar en estudiantes de enseñanza media de la ciudad de Santiago de Chile. In Paz Ciudadana (Org.), Anales del $2^{\underline{o}}$ Seminario de Violencia y Delincuencia. Santiago de Chile: Autor.

Magenzo, A., \& Donoso, P. (2000). "Cuando a uno lo molestan”: un acercamiento a la discriminación en la escuela. Santiago de Chile: LOM. 
Magenzo, A., Toledo, M., \& Rosenfeld, C. (2004). Intimidación entre estudiantes. Como identificarlos y cómo atenderlos. Santiago de Chile: LOM.

Maluf, N., Cevallos, C., \& Cordoba, R. (2003). Enfrentando la violencia en las escuelas: un informe de Ecuador. In M. Abramovay (Org.), Violência na escola. América Latina e Caribe (pp. 251-328). Brasilia: UNESCO.

Mena, I., \& Vizcarra, R. (2001). Algo nuevo bajo el sol: hacer visible el maltrato y posible la esperanza. Psykhe, 1(10), 81-101.

Moscovici, S. (1961). La psychanalyse, son image et son public. Paris: Presses Universitaires de France.

Navarro, L. (2003). Enfrentando la violencia en las escuelas: un informe de Chile. In M. Abramovay (Org.). Violência na escola. América Latina e Caribe (pp. 209-250). Brasilia: UNESCO.

Olweus, D. (1998). Conductas de acoso y amenaza entre escolares. Madrid: Morata.

Ortega, R. (1998) La convivencia escolar: qué es y cómo abordarla. Sevilla: Consejería de Educación y Ciencia.
Ortega, R., \& Mora-Merchán, J. (1997). Agresividad y violencia. El problema de la victimización entre escolares. Revista de Educación, 313, 7-27.

Pikas, A. (1989). The common concern method for the treatment of mobbing. In E. Munthe \& E. Roland (Orgs.), Bullying: an international perspective. Londres: David Fulton.

Strauss, A., \& Corbin, J. (1994). Grounded theory methodology. An overview. In N. Denzin \& Y. Lincoln (Orgs.), Handbook of qualitative research (pp. 273-285). Thousand Oaks, California: Sage.

Taylor, S., \& Bogdan, R. (1986). Introducción a los métodos cualitativos de investigación. La búsqueda de significados. Buenos Aires: Paidós.

Todorov, T. (1995). La vie commune. Paris: Seuil.

Viscardi, N. (2003). Enfrentando la violencia en las escuelas: un informe de Uruguay. In M. Abramovay (Org.), Violência na escola. América Latina e Caribe (pp.153-208). Brasilia, UNESCO.

Zerón, A. (2004). Violencia escolar y violencia anti-escuela: aportes teóricos para una aproximación sociológica. Boletín de Investigación Educacional, 19(2), 43-61.

${ }^{1}$ Este artículo corresponde a un aspecto parcial de los resultados del Proyecto Fondecyt (Fondo Nacional de Investigación Científica y Tecnológica) № 1010771: Hacia una Interpretación de la Violencia en la Escuela. Lineamientos de Estrategias de Convivencia Social en el Espacio Educativo, aprobado para el año 2001. Además se inserta en el marco del Proyecto Fondecyt № 1040694: Figuras Estructurales de la Violencia Escolar. Hacia una Recuperación de la "Subjetividad" Educativa, aprobado para el año 2004.

${ }^{2}$ Quisimos mantener estas diversas categorías, pese a estar referidas unas a las otras de una manera patente, por el simple hecho, de rescatar la diversidad y riqueza del lenguaje de los jóvenes a la hora de explicar sus conductas y la de sus pares. Ser "choro” alude a dos cosas: una es la actitud siempre agresiva y por otra, el "choro” es el ladrón, el que roba (“chorea”).

Mauricio García, doutor em Psicologia pela Universidade Católica de Louvain, Bélgica, é professor em Facultés Universitaires Saint Louis, Bruxelas, Bélgica. Endereço para correspondência: 43, Bvd. du Jardin Botanique; 1000 Bruselas, Bélgica. Tel.: (32)(2) 2117920. Fax: (32)(2) 2117997. E-mail: garcia@fusl.ac.be Pablo Madriaza, mestre em Antropologia e Desenvolvimento pela Universidade do Chile, é psicólogo clínico no Servicio de Salud Metropolitano Norte, COSAM de Conchalí, Santiago, Chile. E-mail: pmadriaz@puc.cl 